\title{
Concept for the controlled plane wave exposure for animal experiments using a parabolic reflector
}

\author{
S. Tejero, S. Schelkshorn, and J. Detlefsen \\ Institute for High Frequency Engineering, Technische Universität München, 80290 München, Germany
}

\begin{abstract}
In this paper a low-cost concept for the controlled RF plane wave exposure for in vivo experiments is presented. The exposure setup is based on the use of a parabolic reflector to convert the incident spherical wavefront emanating from the primary source into a plane wave. The employed paraboloid is a common prime focus paraboloid used in satellite-TV links. The main problems of the focussed approach are identified and a solution based on a defocussed system is introduced. It results in a compact, cost-effective and still power-efficient setup for the RF exposure at microwave frequencies.

Simulation results show a very good performance of the concept achieving a quasi-plane wave incident on the animals with minimum variations of the exposure dose.
\end{abstract}

\section{Introduction}

Together with the great expansion of mobile communications in the last years, the public concern about possible negative effects of these systems on human beings has grown. Although it is impossible in the strict sense to prove the absence of any effect, to get a strong body of evidence for this absence should be enough. However, a body of evidence can only be considered strongly if it is coherent and comprehensive with respect to the raised health concern and not an accumulation of more or less randomly collected negative findings. For our special case of developing an exposure setup, this means that the exposure conditions must be controlled, well defined, standard and repeatable. In this paper a low-cost setup with these characteristics for a specific exposure case is presented. However, the concept can be applied to other setups with different requirements.

The setup is intended for a long-term, continuous and controlled exposure of living Wistar rats. The animals are divided into three different groups: two of them will be exposed, with a GSM- and a UMTS-signal respectively, and one serves as control group, i.e. the animals will have the same environmental conditions but no exposure at all. The isolation between the three groups must be assured to ensure controlled exposition.

Correspondence to: S. Tejero (simon.tejero@tum.de)
To obtain meaningful conclusions from the statistics the number of exposed animals must be high enough, a minimum of 100 rats per setup should be exposed simultaneously. Other requirements are imposed by the in vivo characteristic of the experiment: the exposure chamber must be air-conditioned and the access to the cages must be assured for the daily animal care.

The animals will be exposed by a linear polarized plane wave incident on them. Incident plane wave is the most commonly used exposure condition for in vivo experiments. Of course, the variations of the exposure dose must be kept as low as possible.

To achieve the plane wave condition a long distance from the antenna to the observation point is usually required. That allows to consider the spherical wavefront radiated from the source as a plane or quasi plane wave, i.e. a wave where small deviations from the pure plane wave are tolerated. This approach requires a large exposure setup and is also very inefficient concerning exposure energy, as the signal power density decreases with the square of the distance.

Other energy efficient approaches found in the literature are not suitable for long-term continuous exposure (e.g. Schönborn et al., 2004) or are based on the propagation of guided TEM waves and may run into problems when frequency increases, as the dimensions required to avoid propagations of high order modes are too small (e.g. Hansen et al., 1999; Balzano et al., 2000).

The setup presented in this document is in analogy to the "compact range" concept (e.g. Balanis, 1997), which is used in antenna measurement facilities to obtain a plane wave in relative short ranges. The idea behind is to convert a spherical wavefront into a plane one by using parabolic reflectors. The requirements in antenna measurements are very restrictive in terms of error to a pure plane wave and polarization changes, resulting in big reflector sizes, high distance from the source to the quiet zone - the zone where the plane wave condition is best approximated - and very high price. The approach presented here is based on a common parabolic reflector used in satellite-TV links and results in a compact, cost-effective and still power-efficient setup.

Although the setup described in this paper is intended for a specific experiment, our concept can also be used for other kind of plane wave exposure setups. 


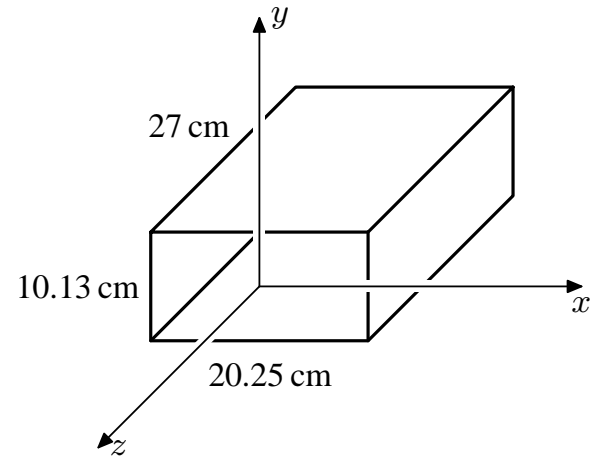

Fig. 1. Open waveguide for the GSM band (925 MHz) operating in the fundamental mode $\mathrm{TE}_{10}$ and radiating $1 \mathrm{~W}$.

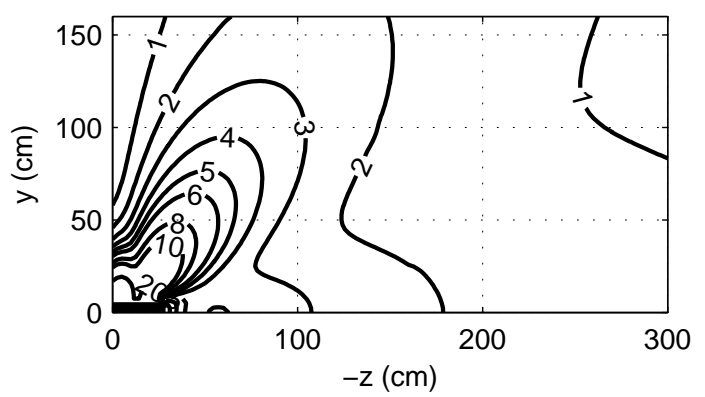

Fig. 2. Field $\left|E_{y}\right|$ (in $\mathrm{V} / \mathrm{m}$ ) of the GSM band ( $925 \mathrm{MHz}$ ) waveguide operating in the fundamental mode $\mathrm{TE}_{10}$ and radiating $1 \mathrm{~W}$ in the absence of the paraboloidal reflector.

\section{Development of the setup}

The principal idea of the exposure concept described here is the well known approach of using a parabolic reflector to convert a spherical wavefront emanating from a primary source located at the focal point into a plane wave. The reflector employed is a common prime focus paraboloid with a diameter of $320 \mathrm{~cm}$ and a focal distance of $112 \mathrm{~cm}$ and originally intended for satellite-TV links. The use of a massproduction good reduces the total cost of the equipment drastically.

Assuming a common prime focus paraboloid illuminated by an ideal source located at its focus, i.e. a point source radiating only towards the reflector and illuminating it uniformly, the reflected wavefront on the paraboloid at the focal plane would be a plane wave with a rather constant electric field and only disturbed by the diffraction caused by the reflector rims. The main problems of this approach are those created by the non-ideal feed. One problem is the spillover effect: a real feed not only radiates towards the reflector, it radiates also towards the cages zone, disturbing the homogeneity of the field distribution. The other main problem is to achieve a uniform illumination of the reflector's surface. An offset paraboloid could be used to avoid these two problems: the exposure zone, located at the focal plane, does not contain the feed and the solid angle covered by the reflector viewed
Table 1. Radiation characteristic of infinitesimally small antennas.

\begin{tabular}{|c|c|c|c|c|}
\hline Antenna & Radiation ch & acterist & and $3 \mathrm{~dB}$-beam & idth \\
\hline & E-plan & & H-plane & \\
\hline Dipole & $\cos \theta$ & $90^{\circ}$ & 1 & $360^{\circ}$ \\
\hline $\begin{array}{l}\text { Aperture in } \\
\text { free-space }\end{array}$ & $(1+\cos \theta) / 2$ & $131^{\circ}$ & $(1+\cos \theta) / 2$ & $131^{\circ}$ \\
\hline $\begin{array}{l}\text { Aperture on a } \\
\text { ground plane }\end{array}$ & 1 & $360^{\circ}$ & $\cos \theta$ & $90^{\circ}$ \\
\hline
\end{tabular}

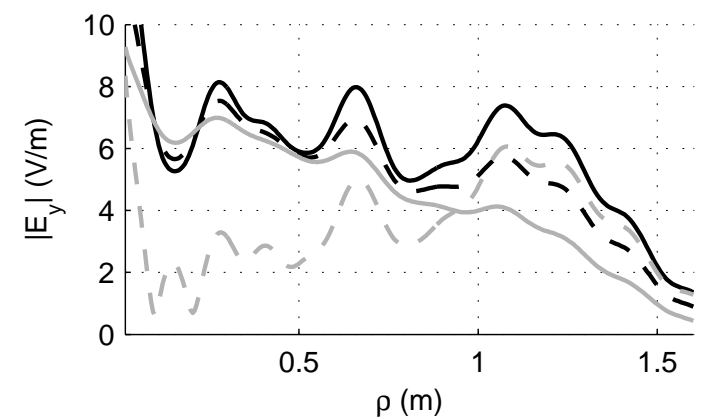

Fig. 3. Electric field at $z=-150 \mathrm{~cm}$ reflected on the paraboloid from Fig. 7 illuminated by a point source located at the focus and radiating in the UMTS band (1966 MHz) with different patterns: uniform (solid black), $(1+\cos \theta) / 2$ (dashed black), $\cos \theta$ (solid gray) and $\sin \theta$ (dashed gray).

from the focus is small. Unfortunately, the low-cost commercially available offset paraboloids have diameters lower than $2 \mathrm{~m}$ and this is not enough to expose a high number of animals.

\subsection{Feed spillover problem}

To identify the region where the field radiated by the primary feed can be neglected a typical wide-beam feed, the open waveguide operating in the fundamental mode, is analyzed. The analyzed waveguides (e.g. Fig. 1) radiate $1 \mathrm{~W}$ and operate in the fundamental mode $\mathrm{TE}_{10}$ in the GSM $(925 \mathrm{MHz})$ and the UMTS (1966 MHz) bands respectively. The simulation for the field is carried out with the Method of Moments $(\mathrm{MoM})$. As shown in Fig. 2 for the GSM band case (and the same is valid for the UMTS one), $150 \mathrm{~cm}$ behind the aperture $(z<-150 \mathrm{~cm})$ the field is 10 times lower than in the vicinity of the aperture and also much lower than the field expected from the reflection on the paraboloid. Hence, it can be stated that the spillover effect of the primary feed becomes negligible for $z<-150 \mathrm{~cm}$. Furthermore, by means of experimental measures, this disturbing field radiated toward the cages by the primary source can be additionally reduced.

Hence, the feed spillover problem will be solved by placing the exposure zone in a region in which the assumption of negligible field of the primary feed is valid: i.e. $2 \mathrm{~m}$ behind the feed aperture, in $-250 \mathrm{~cm} \leq z \leq-200 \mathrm{~cm}$. 


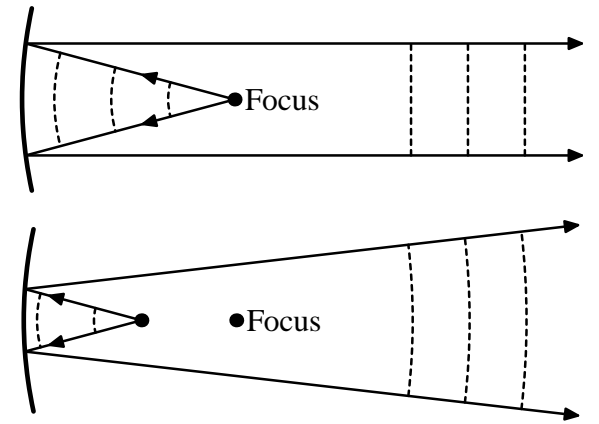

Fig. 4. Reflected wave on a parabolic reflector in a focussed and a defocussed system.

\subsection{Uniform illumination problem}

Another problem of the focused approach is to illuminate the reflector uniformly. As the focal distance-to-diameter ratio is low, i.e. 0.38, the angle which must be uniformly illuminated by the source is very large, i.e. $142^{\circ}$. Comparing this value with the half-power beamwidth of elemental radiators (see Table 1) gives us an idea of the realization difficulty of a source with the desired beamwidth. Also the angle required to illuminate only the region with radius $\rho<125 \mathrm{~cm}$ (the minimum to expose the required number of animals) is rather large, i.e. $116^{\circ}$.

Figure 3 shows the reflected field on the paraboloid from Fig. 7 illuminated with different radiation patterns. It is observed that to obtain a similar homogeneity as with the uniform illumination, the exposure zone radius must be reduced at least to $1 \mathrm{~m}$, not enough to expose a high number of animals.

Also the presence of ripples is observed in the reflected field. These ripples are caused by abrupt changes in the field distribution at the focal-plane aperture (Boonzaaler and Pistorius, 1988). Illumination patterns with a smooth tapering, like $\cos \theta$, prevent these undesired ripples.

To overcome the illumination problem the reflector will be defocussed. As seen in Fig. 4, in a defocussed configuration the reflected wavefront is curved and as the wave propagates the illuminated area increases. The defocussing together with the placement of the quiet zone far enough from the reflector can be used to reduce the primary source's beamwidth requirements. Even though due to the defocussing the wave is not plane any more, the radius of the wavefront is high enough to consider the wave as quasi plane in the region occupied by a cage and to keep the loss in power efficiency within an acceptable limit.

Furthermore, the defocussing generates a smooth tapering of the field at the focal plane preventing the above mentioned undesired ripples in the reflected field.

To obtain the optimum defocussing distance $d_{z}$, some statistical parameters of the electric field in the exposure zone will be analyzed. By means of the Physical Optics (PO) the reflected field on the paraboloid is obtained for different

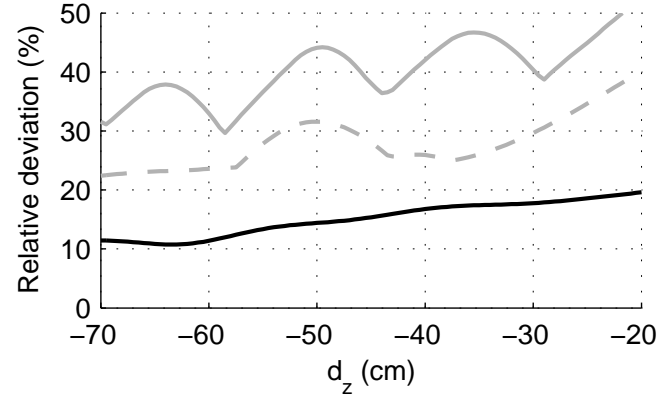

GSM-band setup

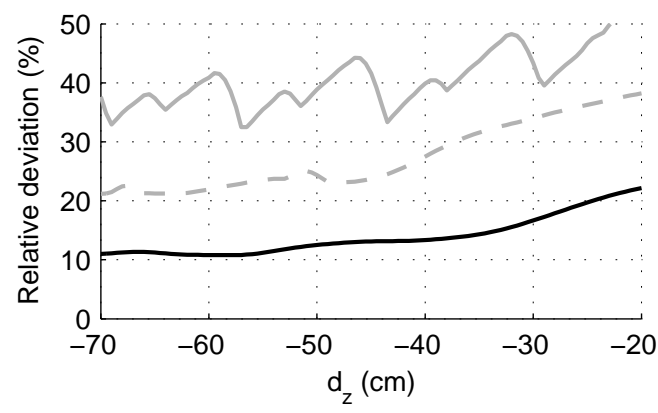

UMTS-band setup

Fig. 5. Standard deviation (black line), maximum positive deviation (gray solid line) and maximum negative deviation (gray dashed line) from the mean value of the reflected field evaluated at the exposure volume as a function of the defocussing distance. The optimum defocussing distances are $-59 \mathrm{~cm}$ and $-56 \mathrm{~cm}$ for the GSMand UMTS-band setups respectively.

$d_{z}$ values in the volume identified for the exposure, i.e. $250 \mathrm{~cm} \leq z \leq-200 \mathrm{~cm}$ and $15 \mathrm{~cm} \leq \rho \leq 125 \mathrm{~cm}$, at the points given by a $4 \mathrm{~cm}$ rectangular grid. With the 10192 field samples, the standard deviation and the maximum positive and negative deviations from the mean value are obtained. Analyzing these parameters (shown in Fig. 5), the optimum defocussing distances has been set to $-59 \mathrm{~cm}$ and $-56 \mathrm{~cm}$ for the GSM- and UMTS-band setups respectively.

\subsection{Proposed approach}

Figure 6 shows the final exposure setup which is placed in a metallic shielded chamber. Besides the paraboloid and the defocussed feed, the absorber material on the walls and the cages for the animals are shown.

To avoid the uncontrolled reflection of the waves propagating in the chamber, absorber material is put at the walls. The absorber material is a high loss material with an impedance comparable to the characteristic impedance of free space, i.e. an incident wave is mainly absorbed and only a small amount is reflected. With typical absorber materials the amplitude of the reflected wave is at least $20 \mathrm{~dB}$ lower than the incident wave.

The exposure volume is the cylindrical region with radius $\rho<125 \mathrm{~cm}$ and depth $-250 \mathrm{~cm} \leq z \leq-200 \mathrm{~cm}$. 


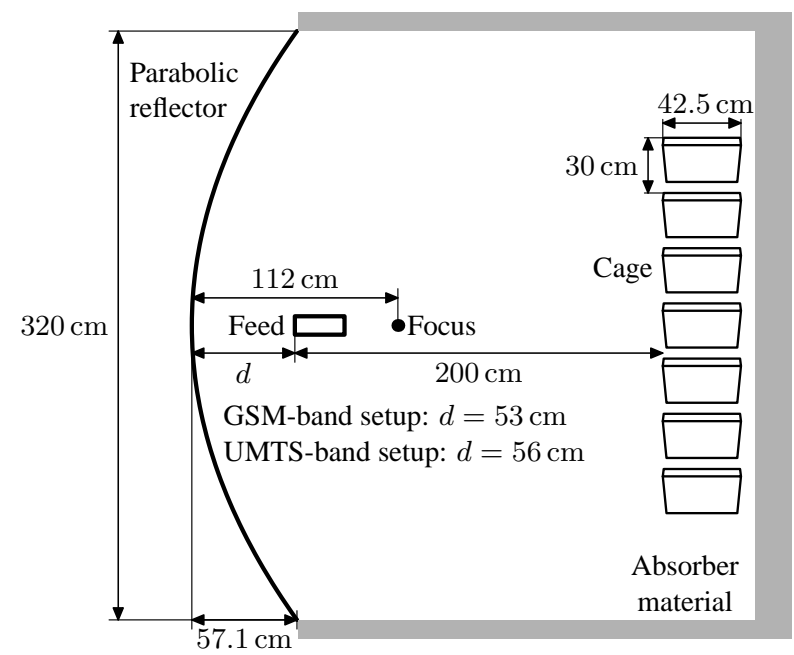

Fig. 6. Side view of the exposure setup.

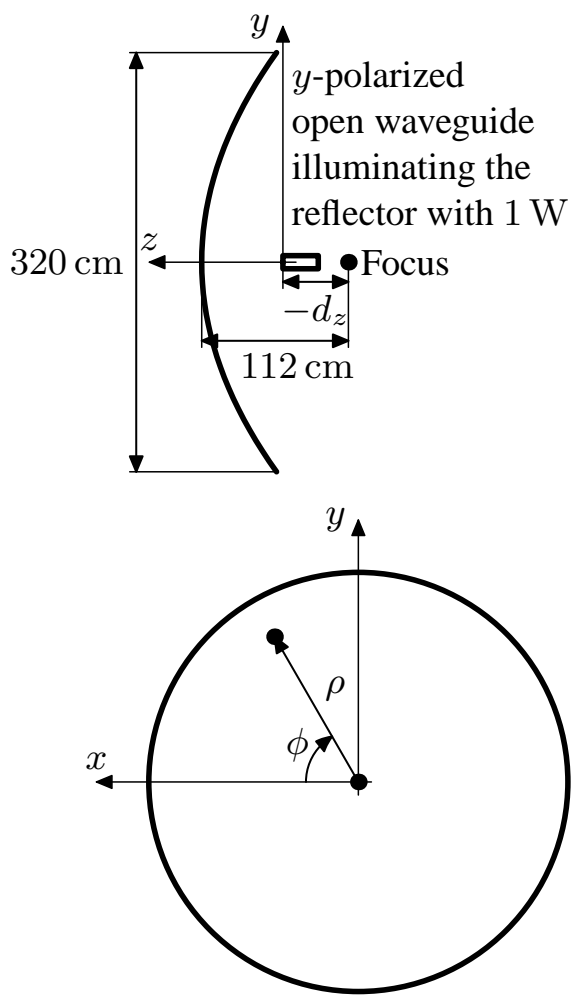

Fig. 7. Simulated exposure setup: $d_{z}=-59 \mathrm{~cm}$ for the GSM-band setup and $-56 \mathrm{~cm}$ for the UMTS-band setup

The animals are inside polysulfan cages with size $(H \times W \times L) 24 \times 28.5 \times 42.5 \mathrm{~cm}^{3}$. The cages are placed on wood slats with the cage frontal looking toward the reflector. The horizontal distance between the cages is $30 \mathrm{~cm}$ and between the slats also $30 \mathrm{~cm}$. As the diameter of the cross section of the quiet zone is planned to be $2.5 \mathrm{~m}$, a large number of cages (approx. 40) can be placed in the setup's quiet zone.
Table 2. Simulation results for the field distribution in the exposure volume.

\begin{tabular}{|c|c|c|}
\hline & GSM Band & UMTS Band \\
\hline Mean value & $7.6 \mathrm{~V} / \mathrm{m}$ & $7.6 \mathrm{~V} / \mathrm{m}$ \\
\hline Standard deviation & $11.2 \%$ & $10.7 \%$ \\
\hline Maximum positive deviation & $33 \%$ & $31 \%$ \\
\hline Maximum negative deviation & $-23 \%$ & $-23 \%$ \\
\hline $\begin{array}{l}\text { Relative frequency of points } \\
\text { with a deviation under } 15 \%\end{array}$ & $76 \%$ & $83 \%$ \\
\hline $\begin{array}{l}\text { Relative frequency of points } \\
\text { with a deviation over } 25 \%\end{array}$ & $0.5 \%$ & $0.5 \%$ \\
\hline $\begin{array}{l}\text { Maximum phase deviation from } \\
\text { a plane wavefront }\end{array}$ & $\pm 12^{\circ}$ & $\pm 12^{\circ}$ \\
\hline Power efficiency & $34 \%$ & $34 \%$ \\
\hline
\end{tabular}

\section{Simulation results}

The simulation for the electric field in the setup is carried out using a combination of two numerical approaches: the Method of Moments (MoM) and the Physical Optics (PO) approximation. The MoM, typically used for electrically small problems, is used to simulate the feed. The PO approximation, a technique to model the electric currents in electrically large objects, is employed for the parabolic reflector.

The final setup's model is shown in Fig. 7: a defocussed paraboloid - with defocussing distance $d_{z}=-59 \mathrm{~cm}$ for the GSM-band setup and $-56 \mathrm{~cm}$ for the UMTS-band setup with a diameter of $320 \mathrm{~cm}$ and a focal distance of $112 \mathrm{~cm}$ illuminated by a $y$-polarized open waveguide operating in the fundamental mode $\left(\mathrm{TE}_{10}\right)$ and radiating $1 \mathrm{~W}$ (see Fig. 1). The field distribution has been calculated at two different frequencies: at $925 \mathrm{MHz}$ (GSM band) and at $1966 \mathrm{MHz}$ (UMTS band).

As stated in Sect. 2, the near field of the open waveguide can be neglected for $z<-150 \mathrm{~cm}$. Therefore, only the reflected component of the total field (the PO component) is investigated.

To analyze the statistics of the field in the exposure zone, the reflected field on the paraboloid is calculated in the exposure volume, $-250 \mathrm{~cm} \leq z \leq-200 \mathrm{~cm}$ and $15 \mathrm{~cm} \leq \rho \leq 125 \mathrm{~cm}$, at the points given by a $2 \mathrm{~cm}$ rectangular grid. With the 80106 field samples a statistical analysis is done. The standard deviation is $11.19 \%$ for the GSM-band setup and $10.70 \%$ for the UMTS-band setup.

The power efficiency - defined as the quotient between the power flowing through the frontal exposure surface and the power fed into the primary feed - is $34.4 \%$ for both setups. Assuming a mean whole-body Specific Absortion Rate (SAR) of $0.025 \frac{\mathrm{W} / \mathrm{Kg}}{\mathrm{W} / \mathrm{m}^{2}}$ for a Wistar rat (Fröhlich et al., 2003) the efficiency in terms of mean whole body SAR per unit of input power (SAR efficiency) is estimated to be $0.002 \frac{\mathrm{W} / \mathrm{Kg}}{\mathrm{W}}$. Assuming that 40 cages with 3 rats each cage will be exposed, a total of 120 rats with an estimated weight 


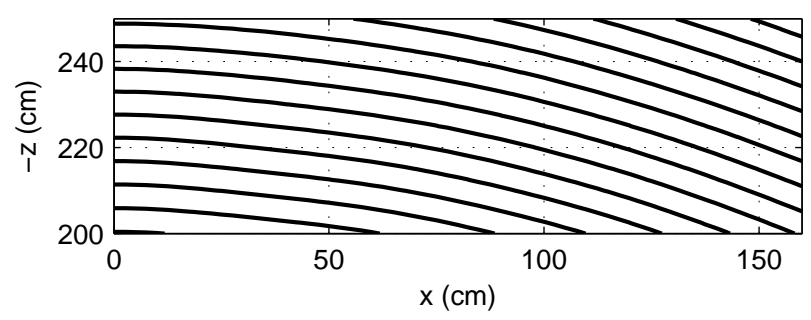

GSM-band setup

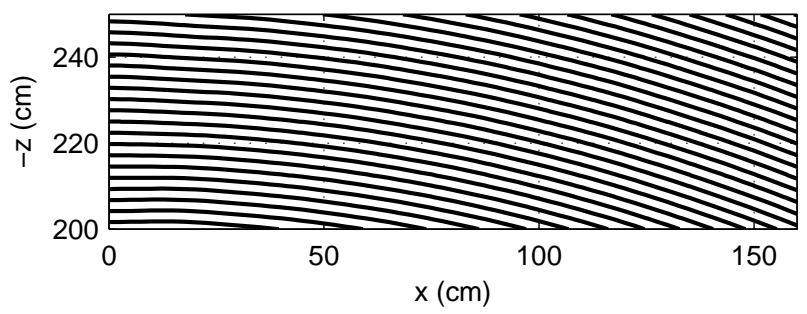

UMTS-band setup

Fig. 8. Phase fronts $\left(\Phi\left(E_{y}\right)=n \cdot 60^{\circ}, n=0,1,2, \ldots\right)$ at the $x z$-plane of the electric field reflected on the paraboloid of Fig. 7. In the region occupied by a cage $\left(W \times H \times L=24 \times 28.5 \times 42.5 \mathrm{~cm}^{3}\right)$ the maximum deviation from a plane phase front is lower than $\pm 12^{\circ}$.

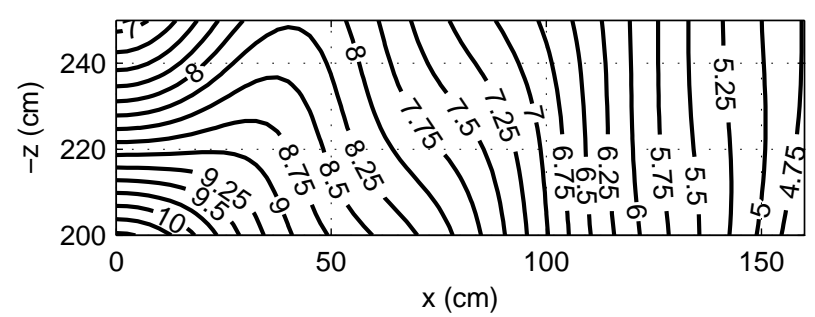

GSM-band setup

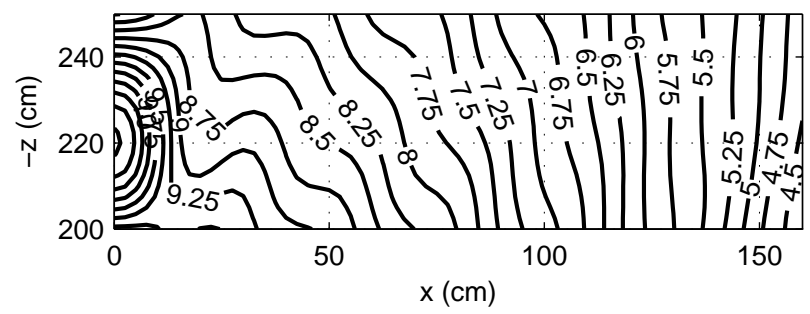

UMTS-band setup

Fig. 9. Absolute value of the electric field (in $\mathrm{V} / \mathrm{m}$ ) reflected on the paraboloid of Fig. 7 illuminated with $1 \mathrm{~W}$. In the exposure volume the standard deviation is $11 \%$.

of $275 \mathrm{~g}$ will absorb $6.5 \%$ of the power radiated by the primary source.

In Fig. 8 the phase fronts at the $x z$-plane are presented. In the region occupied by a cage with dimensions $W \times H \times L=24 \times 28.5 \times 42.5 \mathrm{~cm}^{3}$ the maximum deviation from a plane phase front is lower than $\pm 12^{\circ}$ for both setups.

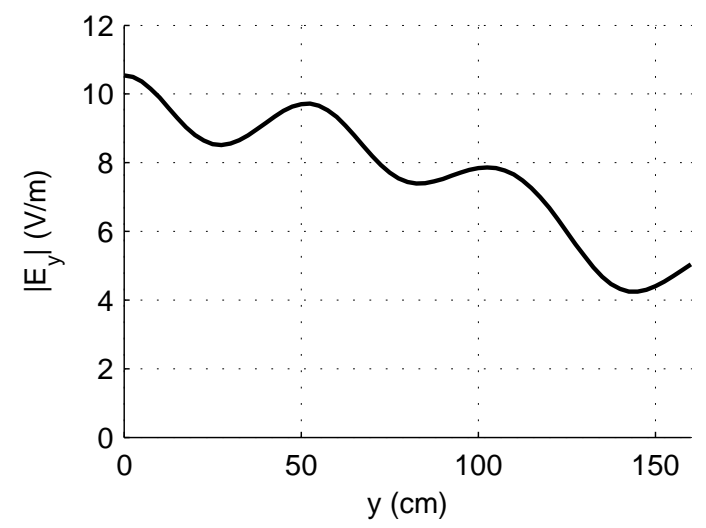

GSM-band setup, $z=-200 \mathrm{~cm}$

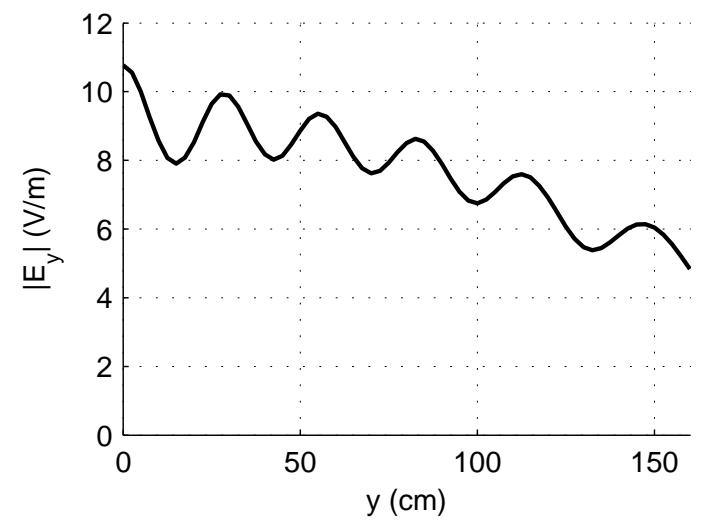

UMTS band $z=-217.5 \mathrm{~cm}$

Fig. 10. Absolute value along the worst case line of the electric field reflected on the paraboloid of Fig.7 illuminated with $1 \mathrm{~W}$. The maximum deviations from the mean value are $+33 \% /-23.50 \%$ for the GSM-band setup and $+31 \% /-23.50 \%$ for the UMTS-band setup.

In Fig. 9 contour plots of the absolute value of the electric field are shown. Figure 10 presents the field along the worst case line, where the field presents maximum variations. The maximum deviations from the mean value are $+33 \% /-23.50 \%$ for the GSM-band setup and $+31 \% /-23.50 \%$ for the UMTS-band setup.

Figure 11 shows the polarization ellipses at the plane $z=-200 \mathrm{~cm}$. In the region with radius $\rho<125 \mathrm{~cm}$ the maximum inclination of the main axis is lower than $3^{\circ}$ for both setups and the axis relation greater than 10 and 17 for the GSM- and UMTS-band setups respectively. Hence, the resulting polarization can be considered linear.

\section{Conclusions and outlook}

A RF exposure setup based on a parabolic reflector is presented. The problems related to a common prime focus paraboloid are solved by defocussing the system and placing the quiet zone away from the feed. A quasi plane wave is generated in a cylindrical region with depth $250 \mathrm{~cm} \leq z \leq-200 \mathrm{~cm}$ and radius $15 \mathrm{~cm}<\rho<125 \mathrm{~cm}$ with a 


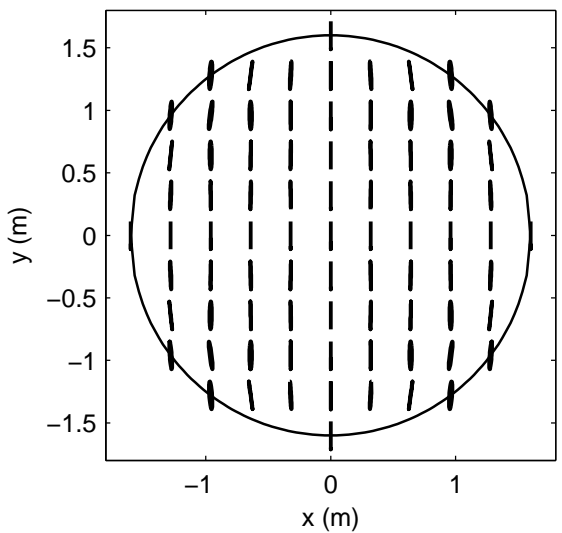

GSM-band setup

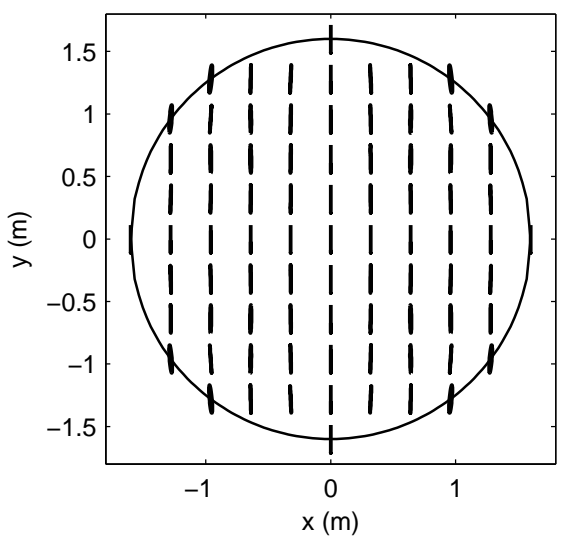

UMTS-band setup

Fig. 11. Polarization ellipses at the plane $z=-200 \mathrm{~cm}$ of the electric field reflected on the paraboloid of Fig. 7. In the exposure region the maximum inclination of the lines is lower than $3^{\circ}$ and the axis relation greater than 10 and 17 for the GSM- and UMTS-band setups respectively. The polarization can be considered linear.

maximum phase error of $\pm 12^{\circ}$ in the volume of a cage and an absolute value standard deviation of $11.2 \%$ and $10.7 \%$ for the GSM- and UMTS-band setups respectively. Within this volume a total of 40 cages with frontal dimensions of $30 \mathrm{~cm} \times 30 \mathrm{~cm}$ can be homogeneously exposed. The power efficiency is approximately $34 \%$ and the SAR efficiency approximately $0.002 \frac{\mathrm{W} / \mathrm{Kg}}{\mathrm{W}}$. The advantages of this concept are the small size of the equipment - a total volume of $3.2 \times 3.2 \times 3.5 \mathrm{~m}^{3}$ is occupied - and the use of a massproduction reflector, which will reduce the total cost of the equipment drastically.

In this paper waves reflected by the absorber material have been neglected and the configuration is still considered without racks, cages and animals, that means without taking into account the mutual effects generated by these objects. Even though the results presented here are expected to be in good agreement with the real field distribution, the final assessment of the exposure will have to take into account all these influences. It is expected that the limits recommended in the literature (Kuster and Schönborn, 2000) will be fulfilled.

\section{References}

Balanis, C. A.: Antenna Theory Analysis and Design, John Wiley \& Sons, New York, 2. edn., 1997.

Balzano, Q., Chou, C.-K., Cicchetti, R., Faraone, A., and Tay, R Y.-S.: An Efficient RF Exposure System with Precise WholeBody Average SAR Determination for in vivo Animal Studies at $900 \mathrm{MHz}$, IEEE Transactions on Microwave Theory and Techniques, 48, 2040-2049, 2000.

Boonzaaler, J. J. and Pistorius, C. W. I.: Degradation of a Plane Wave in a Compact Range, in Antennas and Propagation Society International Symposium, vol. 3, Syracuse, New York, USA, 1402-1405, 1988.

Fröhlich, J., Berdiñas Torres, V., Klingenböck, A., Nikoloski, N., and Kuster, N.: Endbericht Projekt St.Sch4209 "Untersuchung der SAR-Verteilung elektromagnetisch exponierter Versuchstiere", Tech. rep., Bundesamt für Strahlenschutz, 2003.

Hansen, V. W., Bitz, A. K., and Streckert, J. R.: RF Exposure of Biological Systems in Radial Waveguides, IEEE Transactions on Electromagnetic Compatibility, 41, 487-493, 1999.

Kuster, N. and Schönborn, F.: Recommended Minimal Requirements and Development Guidelines for Exposure Setups of BioExperiments Addressing the Health Risk Concern of Wireless Communications, Bioelectromagnetics, 21, 508-514, 2000.

Schönborn, F., Poković, K., and Kuster, N.: Dosimetric Analysis of the Carousel Setup for the Exposure of Rats at $1.62 \mathrm{GHz}$, Bioelectromagnetics, 25, 16-26, 2004. 